\title{
Von Freiberg nach Northampton: Der Weg des Naturheilkundigen Dr. Carl Munde in die USA
}

\author{
Hubertus Averbeck \\ Lilienthal, Deutschland
}

\section{Schlüsselwörter}

Carl Munde $\cdot$ Kaltwasser-Heilanstalt $\cdot$ «Water-Cure» $\cdot$ Hydropathie

\section{Zusammenfassung}

Der Sprachlehrer und Hydropath Dr. phil. Carl Munde wurde durch politische Umstände dazu veranlasst, nach einer bis dahin bunten Karriere seine deutsche Heimat 1849 zu verlassen, um in den USA als Leiter und Besitzer einer Kaltwasser-Heilanstalt, einer sogenannten «Water-Cure», in Florence/Northampton im US-Bundesstaat Massachusetts eineinhalb Jahrzehnte lang sein Glück zu machen. Munde war in Deutschland vor allem durch seine Schriften über die Prießnitzsche Wasserkur und entsprechende eigene Interpretationen bekannt geworden. Auch in den USA wurde sein Name weithin bekannt und ist bis heute mit dem Ortsteil Florence von Northampton verbunden. Munde, der sich als naturalisierter US-Bürger Charles Munde nannte, kehrte nach dem tragischen Verlust seiner Heilanstalt durch einen Brand im Jahr 1866 mit seiner Familie zurück nach Deutschland, um nach einer Zwischenstation in der Schweiz und einer Tätigkeit als Leiter einer Wasserheilanstalt bei Laibach (slowenisch: Ljubljana) schliesslich im damals österreichischen Görz am Isonzo seinen Alterswohnsitz zu nehmen.

() 2015 S. Karger GmbH, Freiburg
Keywords

Charles Munde $\cdot$ Cold water sanatorium $\cdot$ Water-cure $\cdot$ Hydropathy

\section{Summary}

From Freiberg to Northampton: The Path of Naturopath Dr. Carl Munde to the USA

After a hitherto colorful career, the language teacher and hydropath Dr. phil. Carl Munde was forced by political circumstances to leave his German homeland in 1849. He entered the United States of America and tried his luck as the director and owner of a cold water sanatorium, a so-called 'water-cure', in Florence/Northampton, Massachusetts, for one and a half decades. In Germany, Munde had become known especially for his writings on the hydrotherapy of Prießnitz and his corresponding own interpretations. In the USA, his name became also widely known and is still connected to the district of Florence in Northampton. Munde, who called himself Charles Munde as a naturalized US citizen, returned to Germany with his family in 1866 after the tragic loss of his sanatorium due to a fire. After a stopover in Switzerland and a position as director of a hydrotherapy institute in Ljubljana he finally retired in the then Austrian Görz (Gorizia) on the Isonzo river.

(c) 2015 S. Karger GmbH, Freiburg

\section{Einführung}

Der Hydropath Dr. phil. Carl (Gottlieb) Munde, ein aus Freiberg in Sachsen stammender Sprachlehrer, war unter anderem zeitweilig Leiter der Kaltwasser-Heilanstalt (KWHA) Weissenberg bei Freiberg in Sachsen und Direktor einer der ältesten deutschen KWHA, nämlich jener in Elgersburg in Thüringen [1, Titelblatt]. Hingegen ist weniger in die Geschichte der Hydropathie bzw. Naturheilkunde eingegangen, dass er eineinhalb Jahrzehnte in den USA eine solche KWHA, dort als «Water-Cure Estab-

lishment» bezeichnet, zugleich als deren Eigentümer leitete [2; 3, Titelblatt]. Mundes Ruf als erfolgreicher hydropathischer Schriftsteller in Deutschland war ihm offensichtlich in die USA vorausgeeilt. Schon bei seiner Ankunft in Amerika im Jahr 1849 heisst es von ihm, dass er aus der Sicht der Amerikaner der «most eminent water-cure doctor» sei, den Deutschland nach Prießnitz hervorgebracht habe [4]. So schlagzeilenträchtig war sein Erscheinen in verschiedenen Zeitungen wie in der New York Tribune und Baltimore Daily Sun gemeldet worden [5]. Abgesehen davon, dass Munde nach seiner Rückkehr nach Europa 
im Alter von etwas mehr als 70 Jahren in Bad Stein bei Laibach erneut als Leiter einer solchen KWHA berufen wurde, soll in den folgenden Zeilen vor allem verfolgt werden, was Munde dazu veranlasste, seine Heimat zugunsten der USA zu verlassen, was er dort erreichte und weshalb er als naturalisierter US-Bürger nach Deutschland bzw. in die Schweiz und nach Österreich zurückkam.

\section{Munde in Deutschland}

Ausweislich eines Kirchenbuch-Auszugs [6] wurde Carl Gottlieb Munde am 29. März 1805 in Freiberg als viertes Kind des Ratszöllners «unterm Petersthore» Adam Heinrich Gottlieb Munde und dessen dritter Frau Christiane Friedericke geb. Grünbaum geboren. Dies wird in den Arbeiten von Wolfgang Koch [7, S. 32] und Dr. med. Jürgen Fege richtig gebracht [8, S. 643; 9, S. 296], während Sabine Merta in ihrer Dissertation als Geburtsjahr für Munde noch das Jahr 1804 angibt [10].

Munde besuchte von 1815 bis 1819 das Gymnasium seiner Heimatstadt, aus dem er wegen «einer schweren Gesetzesübertretung» relegiert wurde. Er hatte infolge eines «Mangels an Selbstbeherrschung» aus Eifersucht auf die Fenster eines Tanzsaals mit einer Pistole geschossen, weshalb er ausserdem zusätzlich zu einer vierwöchigen Haft in der Fronfeste verurteilt wurde [11]. Nach einer zwischenzeitlich begonnenen Barbierlehre meldete er sich freiwillig zum Militärdienst, aus dem er als Achtzehnjähriger desertierte, um sich zur französischen Fremdenlegion abzusetzen, aus der er 1824 ebenfalls desertierte [7, S. 32]. Auf vielen Umwegen kehrte er zurück nach Deutschland. Wenig später wurde er in Erfurt verhaftet und nach Sachsen wegen seiner Fahnenflucht aus sächsischen Diensten ausgeliefert. Am 29. Juni 1824 verurteilte ihn ein Kriegsgericht zu 25 Stockschlägen sowie 2 Jahren und 8 Monaten «Eisenhaft» [11].

Nach seiner vorzeitigen Freilassung aus der Haft konnte Munde trotz seiner bisherigen unrühmlichen Lebensgeschichte zunächst die Stelle seines Vaters als Zöllner unter dem Peterstor in Freiberg übernehmen. Nebenbei lernte er Französisch sowie zusätzlich Italienisch, Spanisch und vor allem Englisch. Als ergänzender Broterwerb diente ihm die Erteilung von Privatunterricht in diesen Sprachen, wofür er sich ab 1832 empfahl. Bereits im Januar 1826 hatte er seine erste Frau, die Tochter des sogenannten Doppelhäusers Lange, Concordia Friederike Lange, geehelicht. Mit ihr soll er insgesamt neun Kinder gehabt haben [11], was jedoch so nicht zutreffend zu sein scheint, wie sich aus seinem Testament von 1880 ergibt (Mitteilung an den Autor - Kopie im Besitz von Dr. J. Helfricht, Radebeul). Munde betrieb nebenberuflich ab März 1830 eine Lotteriekollektion und eine Kaffeewirtschaft mit Billard. Schon bis 1833 hatte er seinen ange- schlagenen Ruf wieder so weit hergestellt, dass er zur Unterstützung des Professorenkollegiums der Bergakademie in Freiberg als Sprachlehrer eingesetzt wurde. Wenn auch gegen den Willen eines Teils der «rechtschaffenen Bürgerschaft» Freibergs, bei der seine Jugendtorheiten unvergessen waren, erhielt er dennoch in der Akademie im Oktober 1833 auf Veranlassung des Oberberghauptmanns Siegmund August Wolfgang Freiherr von Herder (17761838) zwar nicht die erhoffte Stellung als akademischer Lehrer, sondern zu seinem Leidwesen nur die als «Nebenlehrer» für Französisch zum Jahresgehalt von 100 Thalern. Aus dieser Zeit an der Bergakademie resultieren auch Mundes erste Lehrbücher für Englisch und Französisch, die in mehreren Auflagen erschienen. Die Stellung an der Bergakademie hatte er mit Unterbrechung (Freistellung) bis zu seiner von ihm selbst erbetenen Entlassung im Jahr 1841 inne. Zusätzlich gründete er im Jahr 1838 eine antiquarische Buchhandlung, eine Volks- und Jugendbibliothek sowie eine private Turnanstalt in Freiberg. Dennoch spricht er später kurz vor seiner Übersiedelung in die USA in einem Brief vom 25.07.1849 an den US-Diplomaten in Belgien Thomas G(reen) Clemson (1807-1880) davon, dass er Professor an der Bergakademie in Freiberg gewesen sei [12, S. 7-8]. Diesen Brief schickte Clemson an den US-Politiker John C(aldwell) Calhoun senior (17821850) mit wohlwollender Kommentierung und der Bitte, dass unter anderem der Sohn John Calhoun junior sich in den USA für Munde verwenden möge [12, S. 4].

Ein unter anderem seit einiger Zeit bestehendes «Kopfgichtleiden» hatte Munde, der selbst seinen Gesundheitszustand seit Längerem als zerrüttet bezeichnet hatte, was von seinem Sohn Paul Fortunatus (1846-1902) später bestätigt wird [13], veranlasst, sowohl medizinische als auch homöopathische Heilversuche zu unternehmen. Als alle Massnahmen jedoch wirkungslos geblieben waren, suchte und fand er Hilfe erstmals im August 1836 in Gräfenberg bei Freiwaldau im österreichischen Teil Schlesiens bei dem inzwischen schon einigermassen bekannten medizinischen Laien und wasserheilkundigen Landwirtssohn Vincenz Prießnitz (1799-1851). Bereits im Winter $1836 / 37$ «als geheilt entlassen» $[14$, S. $248 ; 15$, S. 325], befasste er sich neben seinen linguistischen Lehrbüchern fortan mit der Prießnitz'schen Kaltwasserkur in Gräfenberg. Zu seinen bedeutenden Schriften aus dem Bereich der Hydropathie bzw. Hydrotherapie gehören neben der «Beschreibung von Gräfenberg» [16] und dem dort praktizierten Kaltwasserkurverfahren vor allem seine sogenannten «Memoiren eines Wasserarztes» in zwei Bänden (1844; 2. Auflage: 1847) [1] sowie die sehr positiv rezensierte Übersetzung und Bearbeitung der 53-seitigen Schrift von Dr. med. Louis Sauvan über die «Darstellung der wissenschaftlichen Prinzipien der Wasserheilkunst oder der sogen. Gräfenberger Heilmethode» [17, 18]. Neben zahlreichen anderen Veröffentlichungen, wie seiner 
englischsprachigen Arbeit von 1857 über das Scharlachfieber [19], in der er über seine 21-jährigen Erfahrungen berichtet, später über die Diphterie (1880) [20] und die Haut (1885) [21], gehörte nicht zuletzt sein Hauptwerk «Hydrotherapie» dazu, das immerhin dreizehn Auflagen allein nur in deutscher Sprache erlebte, deren letzte allerdings posthum von dem Dresdener Arzt Dr. Arthur Pollack überarbeitet herausgegeben wurde [3].

Seit dem Ende der 1830er-Jahre gehörte Munde durch seine Veröffentlichungen zum aktiven Kreis der «Wasserärzte» über Sachen hinaus, sodass ihm sogar - durch Prießnitz veranlasst - die Leitung einer KWHA bei Prag vermittelt wurde. Dieses Angebot hatte er zwar noch abgelehnt, aber seine inzwischen erworbenen theoretischhydropathischen Kenntnisse und Erfahrungen in der Praxis veranlassten ihn schliesslich im Jahr 1838, selbst eine KWHA zu gründen. Deren Eröffnung erfolgte unter der offiziellen Aufsicht unter und zusammen mit dem Bezirksarzt und Freimaurer Dr. Gustav (Adolph Theodor) Ettmüller (1808-1881) im Mai des folgenden Jahres in einer umgebauten, nicht mehr gangbaren Mühle unweit des Ortseingangs von Weissenborn im Muldental bei Freiberg. Diese sogenannte «Langenrinner Mühle» - das Gebäude ist noch heute erhalten -, in der Literatur meist als Kalt-/Wasserheilanstalt in Freiberg bezeichnet, war im Besitz des Müllermeisters Drechsler [22, S. 90]. Mit diesem hatte jedoch Munde in der Folgezeit immer wieder Schwierigkeiten. Unabhängig davon, dass sich seine Heilanstalt wirtschaftlich nicht so positiv entwickelte, wie er sich das vermutlich erhofft hatte [1, Bd. 2, S. 17], war Munde auch mit den Wohnverhältnissen sehr unzufrieden. Insofern war es wenig verwunderlich, dass er eine ihm angebotene Stellung als herzoglicher Bade-Direktor an einer der ersten deutschen KWHA, jener in Elgersburg in Thüringen, annahm [2, S. 360]. Sein Verhältnis zu dem bisher alleinig tätigen ärztlichen Leiter der Elgersburger Anstalt, Dr. med. Hermann Piutti (1812-1865), und schliesslich auch zu dem Eigentümer der Anstalt, Jacob Gräser (1781-1858), war bereits kurz nach der im August 1840 erfolgten hoheitlichen Übertragung seiner neuen Stellung, die er im September 1840 antrat, deutlich gestört [2, S. 362]. Letztlich gab Munde bereits nach knapp einem Jahr auf und verliess Elgersburg wieder. Unabhängig davon hatte er sich durch seine zahlreichen hydriatischen Schriften bereits in den eingeweihten Laienkreisen von an der Hydropathie Interessierten eine erhebliche Anerkennung erworben, jedoch fehlten ihm vermutlich diplomatisches Geschick in der Praxis und die Anerkennung durch die Medizin. Da er aber kein einschlägiges Studium vorzuweisen hatte, ist seine Bemühung um die Erlangung des philosophischen Doktortitels vom 12. Mai $1840 \mathrm{zu}$ verstehen. Wenngleich andernorts die Rede vom Sommer 1841 ist [7, S. 33], erwirbt er laut Promotionsurkunde bereits am 25. Mai 1840 in Jena den akademischen Grad

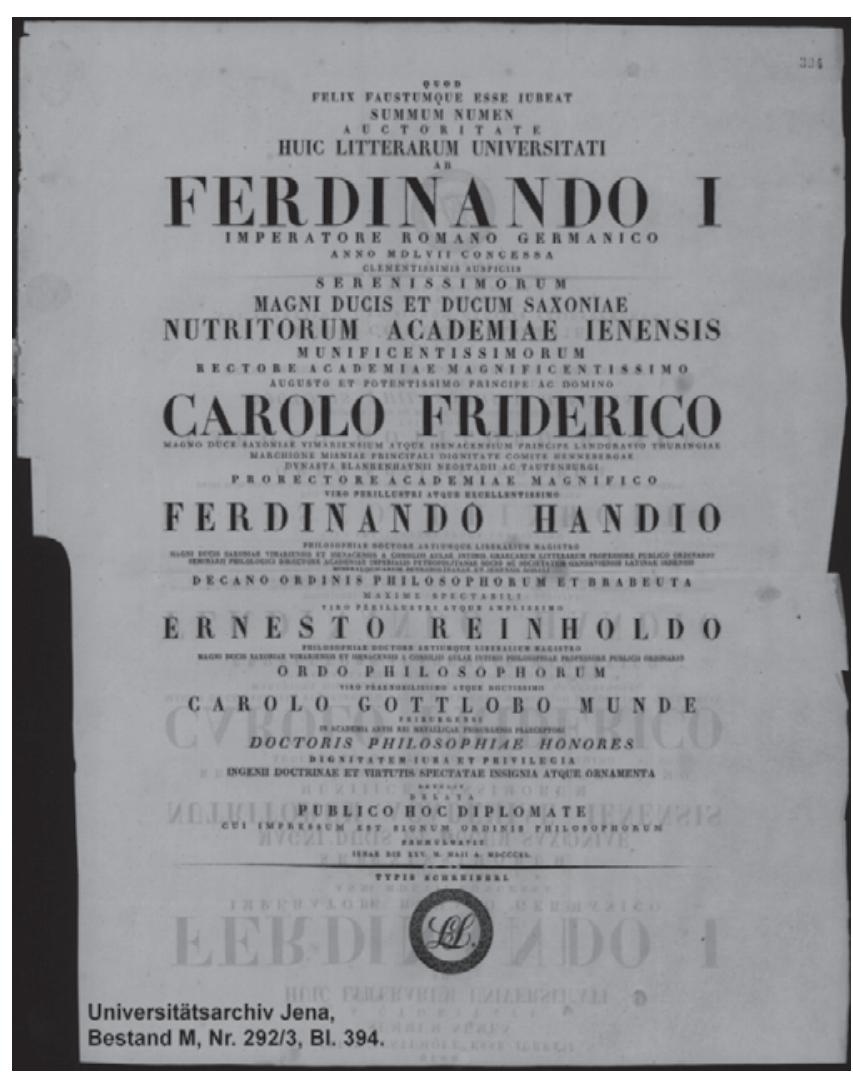

Abb. 1. Doktor-Diplom der Universität Jena für Carl Munde vom 25. Mai 1840. Original im Universitätsarchiv Jena (UAJ, Best. M, Nr. 292/3).

eines «Doctoris Philosophia Honores» (Dr. phil.) (Abb. 1). Auf dieser Urkunde wird sein zweiter Vorname nicht mit Gottlieb, sondern mit Gottlob angegeben. Dem Promotionsverfahren ging ein teilweise erhaltener Schriftwechsel des Mineralogen Professor (August) Breithaupt (17911873) aus Freiberg und dem Jenenser Chemiker (Johann Wolfgang) Döbereiner (1780-1849) sowie die Ausstellung eines Sittenzeugnisses der Stadt Freiberg voraus (persönliche Auskunft UAJ vom 29. Mai 2013, UAJ, Best. M, Nr.292/3, Bl. 386r).

\section{Mundes Stellung vor seiner Emigration}

Nach dem schlesischen Landwirtssohn und Vater der KWHA, Vincenz Prießnitz, gehörte Carl Munde (Abb. 2) unstrittig zumindest in Deutschland neben Dr. phil. Eucharius Ferdinand Christian Oertel (1765-1850) sowie weiteren aktiv publizierenden Hydropathen wie Heinrich Friedrich Fran(c)ke alias Johann Heinrich Rausse (18051848) und später dem schlesischen Fuhrmann und Nachbarn von Prießnitz, Johann Schroth (1798-1856), zu den bekanntesten deutschen Laienhydropathen des frühen 19. Jahrhunderts. Munde kann als einer der Hauptmultiplikatoren bezeichnet werden, die vor allem das neuartige Prießnitz'sche Kaltwasserkurverfahren durch ihre Veröf- 


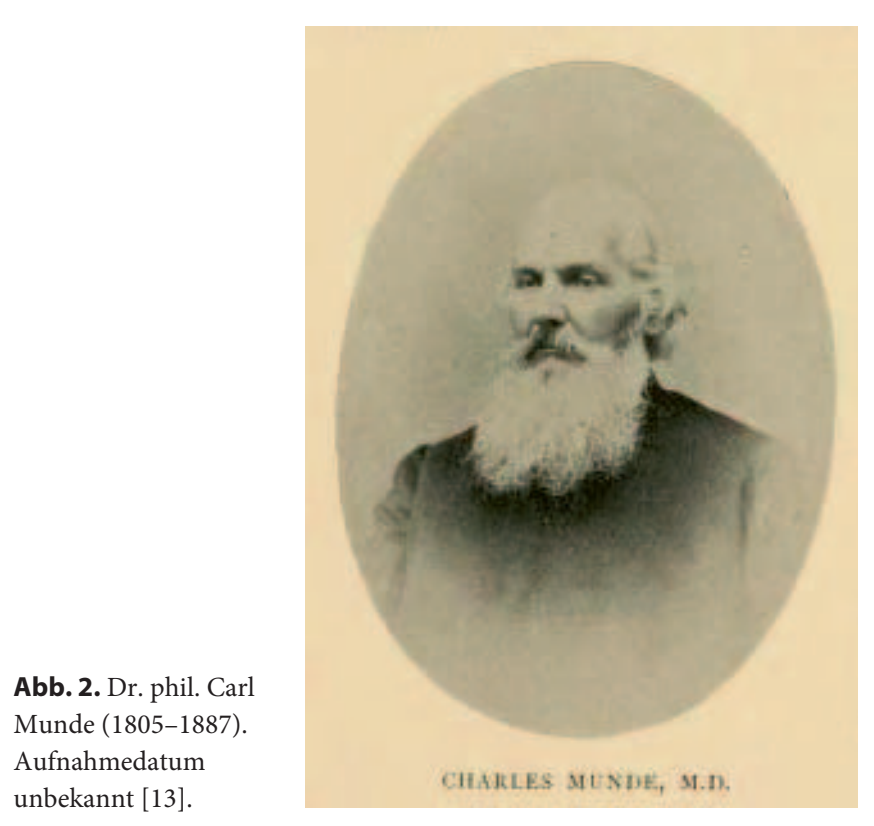

fentlichungen bekannt gemacht und zu deren Durchbruch verholfen haben. Nach Ansicht einiger der zeitgenössischen Autoren haben vor allem medizinische Laien wie Rausse (und Munde) das leitende Prinzip des hydropathischen Verfahrens mit erarbeitet [23, S. 14], derweil ja Prießnitz erfolgreich praktizierte, selbst nicht publizierte und sich auch wenig um einen theoretischen Unterbau seines Verfahrens kümmerte. Inwieweit jedoch das publizistische Tun insbesondere dieser beiden Autoren, Munde und Rausse, tatsächlich eine nennenswerte Auswirkung gerade auch auf die deutsche Ärzteschaft jener Zeit gehabt hat oder ob Ärzte wie etwa neben dem Tierarzt in Freiwaldau, Johann Joseph Weiß (1795-1847), ein Kröber, Plitt, Lauda, Kreyser, Richter, Weigersheim, Haidenheim, Steudel, Petri, Gleich und andere mehr dazu beigetragen haben, wurde sehr unterschiedlich bewertet und wäre es durchaus wert, heute noch zum Gegenstand einer weiteren Diskussion an anderer Stelle gemacht zu werden. Dabei darf aber auch nicht übersehen werden, dass sich später ein Arbeitskreis bildete, an dem gleichermassen Laien wie Mediziner beteiligt waren. Zumindest schreibt Munde im Jahr 1861, dass z.B. seine Arbeit «Hydrotherapie» (ed 10) zwar für Nichtärzte geschrieben wurde, er aber hoffe, dennoch später eine die Ärzte befriedigende Ausgabe fertigstellen zu können [24, S. V]. Unstrittig war Munde ein Multitalent, denn er war nicht nur Sprachlehrer, aktiver Hydropath, kurzzeitig sogar Herausgeber des hydropathischen Journals «Der Wasserfreund» und «Revolutionär», sondern er war vorrangig ein überaus erfolgreicher Schriftsteller vor allem in Sachen Wasserheilkunde, wenn man nur die Zahl der Auflagen seiner Bücher zugrunde legt. Dessen ungeachtet war er vor allem als solcher aus verschiedenen Gründen nicht unumstritten. Munde wird in der Literatur gelegentlich sogar als «Re- negat» bezeichnet [14, S. 247] und hat sich posthum vor allem die Kritik des Prießnitz-Enthusiasten, schlesischen Heimatdichters und Nietzsche-Kenners Philo vom Walde (1858-1906) alias Johannes Reinelt zugezogen [25, S. 57 59]. Dies offensichtlich deshalb, da er in seinen zahlreichen hydriatischen Schriften auch die Vertreter der Wasserkur, ja selbst seinen grossen Lehrer in Sachen Hydropathie, Vincenz Prießnitz, keinesfalls schonte, sondern mit deutlich kritischen Worten auf seiner Ansicht nach vorhandene therapeutische Missstände und Fehler im Bereich der Hydropathie hinwies. Das hat allerdings Rausse ebenso getan, aber nach dessen frühem Tod in Bad Alexandersbad stand er trotz der weiterführenden Aktivitäten seines in die Schweiz emigrierten und sich auf den Vegetarismus konzentrierenden Schülers und Neffen Theodor Hahn nicht mehr so im Mittelpunkt der sich in den 1850er-Jahren erheblich streitenden Szene.

\section{Mundes Emigration in die USA}

Nach dem gescheiterten Engagement in Elgersburg war es zunächst Mundes Absicht, sich nunmehr der Medizin in Form eines Studiums zu widmen. Diese Hoffnung wurde jedoch durch ein längeres Krankenlager von Herbst 1841 bis Sommer 1842 letztlich zunichte gemacht und verhinderte alle weiteren Ambitionen in dieser Richtung. So entschloss sich Munde, Freiberg von 1843 auf 1844 endgültig zu verlassen [7, S. 33]. Munde übernimmt im Jahr 1844 die Stellung des Direktors der Handelslehranstalt in Dresden. Zugleich entwickelt er rege politische Aktivitäten. Die Wasserheilkunde verliert er allerdings auch in dieser Zeit nie aus dem Auge, wie auch seine weiteren Veröffentlichungen und die zeitweilige Mitgliedschaft im bereits 1835 gegründeten «Hydrodiätetischen Verein» von Dresden zeigen [26, S. 10-13].

Bis zu den Jahren der deutschen revolutionären Ereignisse und Einigungsbestrebungen um 1848 hatte er eine Privatpension eröffnet; ausserdem war er Stadtverordneter und in dieser Eigenschaft Mitglied des Ausschusses der Dresdener Bürgerwehr und Mitglied des (radikal-demokratischen) Vaterlandsvereins [7, S. 33]. Darüber hinaus engagierte er sich im örtlichen Turnerverein und war Kommandant der gesetzlich organisierten Turner-Waffenschar. Sein Handeln in diesen Jahren zeigt, dass Munde auf seine Weise ein entschlossener und glühender «Freiheitskämpfer» im Sinne der Revolutionäre von 1848/49 gegen die bestehenden Herrschaftsverhältnisse im Deutschen Bund war. Die militärischen Auseinandersetzungen des 5. Mai 1849 in Dresden führten für Munde $\mathrm{zu}$ einem folgenschweren Ereignis. Er hatte unweit des Stadtschlosses eine Rede an die an den Barrikaden lagernden Soldaten gehalten, geriet auf dem Rückweg nach Hause unter Beschuss und wurde am Unterschenkel des lin- 
ken Beins schwer verletzt. Nachdem er sich einige Tage vor den Schergen der sächsischen Regierung in Dresden verborgen hatte, entkam er mithilfe von Freunden mit seiner zweiten Frau mit ihrem «dritthalbjährigen» Kinde (evtl. Paul Fortunatus, geb. 1846) und seinem 15-jährigen Sohn Albert aus erster Ehe aus der Stadt Dresden zunächst nach Tharandt. Laut einem von Munde in Brüssel geschriebenen Brief vom Juli 1849 handelte es sich bei seiner Frau («Mrs. Munde») um Bertha von Hornemann [12, S. 7], die - wie er hervorhob - neben Deutsch auch sehr gut Englisch sowie Französisch sprach und eine $\mathrm{Mu}$ sikausbildung hatte. Auf vielen Umwegen, die er später sehr eindrucksvoll in zwei längeren Aufsätzen in der Zeitschrift «Der Grenzbote» beschreibt [3], gelangte er schliesslich nach Antwerpen. Munde, der ursprünglich nach England emigrieren wollte, wurde sogar in Belgien ein Lehrauftrag an der Universität Lüttich angeboten, was er auf Anraten von Freunden wegen der dort befürchteten Dominanz der Jesuiten ablehnte, woraufhin seiner Familie und ihm von seinen Freunden die Überfahrt in die USA ermöglicht wurde.

\section{Munde als Leiter einer Kaltwasser-Heilanstalt ("Water-Cure») in den USA}

Durch seine abenteuerliche Flucht aus Deutschland und die Reise nach Übersee [27] konnte er einer Inhaftierung - anders als etwa ein Fritz Reuter - entgehen. Mit vergleichbaren Schicksalen sahen sich nicht nur der erwähnte Jenenser «aufrührerische» Ur-Burschenschafter [28], deshalb jahrelang inhaftierte und mit Berufsverbot belegte Jurist Robert Wesselhöft, dessen väterliche Familie mit dem Druckereibesitzer Johann Carl Wesselhöft (1768-1848) während dessen Weimarer Zeit in enger freundschaftlicher Beziehung zu Johann Wolfgang von Goethe stand [29, S. 250], sondern auch dessen 2 Jahre älterer Bruder, der Chirurg, Baseler Augenarzt und Prosektor Dr. Wilhelm Wesselhöft (1794-1858) konfrontiert. Dieser war ebenfalls Aktivist in der frühen Burschenschaftsbewegung sowie in dem im Untergrund tätigen verbotenen «Jünglingsbund» und dem jedoch nie wirklich zu Bedeutung kommenden «Männer-Bund» der sogenannten «Demagogenverfolgung» in den 1820/30er-Jahren ausgesetzt [30]. Die Brüder Wesselhöft (Robert, der 1843 in Basel promoviert wurde, und vor allem Wilhelm alias William) spielten in den USA insbesondere bei der Einführung der Homöopathie eine nicht unbedeutende Rolle [31], wie unter anderem auch ihre verschiedenen Schriften zeigen.

Bereits kurz nach seiner erfolgreichen Flucht aus Dresden und der Ankunft in New York meldete die New York Tribune in einem längeren Beitrag, der vom «Water-Cure Journal and Herold of Reforms» (WCJ) übernommen

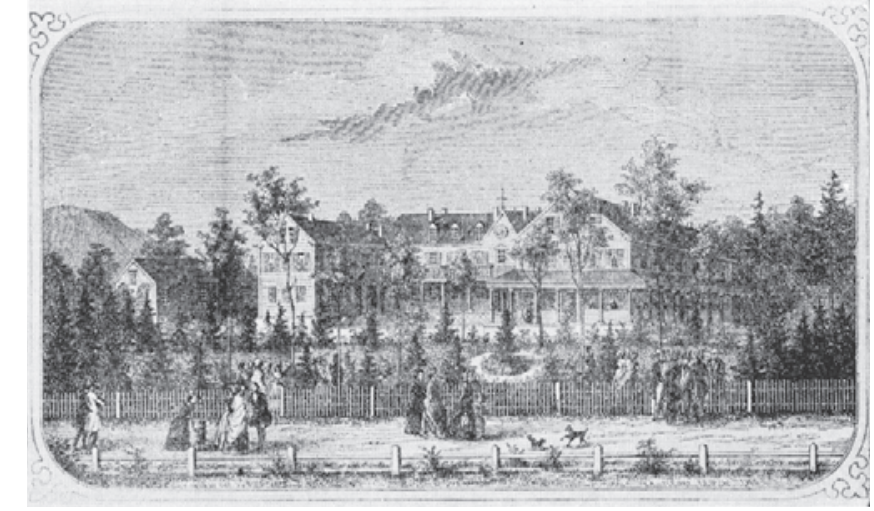

Abb. 3. «Dr. Charles Munde Water-Cure» in Florence, MA, am Mill River [13].

worden war, dass Munde sich als erfolgreicher Hydropath und «physician» (Arzt!) in Baltimore niederlassen wollte [5, S. 186-187]. Diesen ihm vorauseilenden guten Ruf hatte er nicht nur seinen in englischer Sprache erschienenen hydriatischen Schriften zu verdanken, sondern auch der Tatsache, dass zahlreiche angloamerikanische Hydropathen (Laien und Ärzte) durch ihre Herkunft oder ihren Aufenthalt in Deutschland mit seinem Namen vertraut waren. Munde und seine Familie gehörten im Jahr 1849 zu den immerhin 63148 Einwanderern aus den deutschsprachigen Ländern [32, S. 432].

Bereits im Jahr 1850 kommt Munde erstmals in den Northamptoner Vorort mit dem ursprünglichen Namen «Broughton Meadow», der später «Bensonville» und seit 1850 «Greenville» genannt wurde und der für die kommenden nahezu eineinhalb Jahrzehnte unter dem Namen «Florence» sein neues Zuhause werden sollte. Der Ortsname Florence wurde aufgrund der besonders schönen Lage dieses Vororts von Munde selbst in Erinnerung an die Schönheiten des italienischen Florenz anlässlich einer abermaligen Namensfindung im Jahr 1852 vorgeschlagen und von der Mehrheit der Verantwortlichen angenommen; er wurde bis heute beibehalten. Mundes Vorschlag hatte sich gegen andere Vorschläge wie «Leeds» bzw. «Shepherd's Hollow» durchgesetzt [33, S. 107], nicht jedoch seine andere vorgeschlagene Umbenennung des lokalen Gewässers «Mill River» in «Arno». An diesem Mill River lag sein künftiges Arbeitsfeld, die vormalige KWHA («Water-Cure») des verstorbenen David Ruggles (Abb. 3). Bereits im Mai 1850 wird in der vom Arzt Prof. Joseph R. Buchanan herausgegebenen Zeitschrift «Eclectic Medical Journal» darauf hingewiesen, dass der bekannte Hydropath Dr. Munde aus Deutschland die schöne Heilanstalt des verstorbenen Ruggles erworben habe [34].

Hintergrund seiner Reise nach Northampton war, dass Freunde ihm nahegelegt hatten, die von dem Afroamerikaner und Anti-Sklaverei-Aktivisten David Ruggles (1810-1849) um 1842 gegründete KWHA [35-37], die seit dessen Tode verwaist war, zu übernehmen. Es kann als 
gesichert bezeichnet werden, dass sich Munde und Ruggles niemals persönlich begegnet sind. Dies ergibt sich aus den Erinnerungen eines Sohns von Munde, Dr. med. Paul F. Munde (1846-1902), eines namhaften Gynäkologen in New York [13, S. 190-195]. Munde und Ruggles verbindet dennoch nicht nur, dass sie in derselben Heilanstalt, einer sogenannten «Water-Cure», nacheinander tätig wurden, sondern dass sie wie zahlreiche andere ihrer Zeitgenossen durch ihre eigenen gesundheitlichen Leiden zu Anhängern und Vertretern der Naturheilkunde im Allgemeinen und der Kaltwasserkur im Besonderen wurden. Ruggles' hydropathische Aktivitäten hatten dazu geführt, dass «Dr. David Ruggles» vom Herausgeber des Boston Liberator als der «Priessnitz of America» in doch sehr überzogener Weise bezeichnet wurde [5, S. 14], wie dies zuvor schon in einem früheren Artikel im WCJ der Fall gewesen war (vgl. WCJ., Bd. 4, 1847, Nr. 6, S. 357). Munde selbst bezeichnete sich in seinen Werbeanzeigen in den USA als den ältesten noch aktiv tätigen Schüler von Prießnitz. Beide hatten zumindest zeitweilig eine deutliche Verbesserung ihres Krankheitszustands durch Massnahmen jeweils eines deutschen Laienheilkundigen gefunden: Munde bei Vincenz Prießnitz in Gräfenberg in Schlesien, als dessen Schüler er zugleich bezeichnet werden kann, und Ruggles durch den schon erwähnten ehemaligen Juristen und nunmehrigen Homöo-Hydropathen (Dr.) Robert Wesselhöft (1796-1852) in Cambridge, Massachusetts.

Schon kurz nach der Übernahme dieser vormaligen Ruggles'schen KWHA in Bensonville, wie Munde selbst den Vorort bei Northampton auch noch nannte, veröffentlichte er seinen ersten Aufsatz im führenden US-Journal für Hydropathie über die Nutzung des kalten Wassers [38]. In diesem Journal, dem WCJ, machte er auch Werbung für seine KWHA und beschrieb unter anderem deren günstige Lage sowie die Zielsetzung und Mittel seiner Behandlung. Er berechnete für einen Aufenthalt pro Woche und Person 10 USD und für die erste Konsultation 5 USD [38, S. 103]. Frühzeitig engagierte er sich im «Committee of Hydropathy», das unter der Leitung des vielseitig tätigen Vorsitzenden, des Arztes Seth Rogers, M.D., Leiter eines hydropathischen Instituts in Worcester, Massachusetts, stand. Neben diesem gehörten ein Henry Foster in Clinton Springs, New York, und eben Charles Munde, M.D., in Northampton, Massachusetts, dazu. Von diesem Komitee wurden regelmässige Mitteilungen als sogenannte «reports» herausgegeben (vgl. WCJ, Vol. XV, 1853, Nr. 4, S.93).

Munde, der sich sowohl schon 1851 als M.D. als auch 1853 und fortan häufig als Doktor der Medizin und Philosophie bezeichnet hatte [3, Titelblatt], soll in den USA wegen seiner heilpraktisch-medizinischen Tätigkeit die Würde eines «Dr. med. h.c.» verliehen worden sein $[7, S$. $34 ; 9$, S. 644]. Wenngleich dieser Doktortitel kein typisch US-amerikanischer ist, der nämlich «M.D.» (medical doc- tor) lautet, nennt Munde sich in einer Anzeige für seine Wasserheilanstalt im WCJ auch im Juni 1854 [39, Bd. 17, 1854, N. 6, S. 138] ebenso wie im April 1855 «Charles Munde, M.D.» [39, Bd. 19, 1855, Nr. 4, S. 90] und gleichermassen in seinem englischsprachigen Buch über das Scharlachfieber [19, Titelblatt]. Es liessen sich jedoch keine sicheren Nachweise - auch nicht in Mundes Schriften - dafür finden, welche amerikanische Universität, welches College oder welches sonstige Institut ihm wann diesen medizinischen Doktortitel ehrenhalber verliehen haben soll, abgesehen von der Tatsache, dass sich Munde selbst frühzeitig wie oben genannt bezeichnete und seit dem Brendel-Artikel in der «Heimatwarte» von 1939 in einigen deutschen Veröffentlichungen die Rede vom «Dr. med. h.c.» ist [11]. Auch der erwähnte Schieferdecker, ebenfalls ein Schüler von Prießnitz, nannte sich in den USA «M.D.», obwohl er überhaupt keinen «medical degree» hatte [40, S. 88].

Wie aus einem Schreiben des US-Aussenministeriums an die US-Gesandtschaft in Wien vom 28. Juli 1887 hervorgeht und somit als gesichert bezeichnet werden kann, wurde Munde bereits im Jahr 1854 zum US-Bürger naturalisiert und hatte am 21. Mai 1885 erneut einen US-Pass erhalten.

Bis zum Jahr 1849 hatte es in den USA bereits 16 KWHAs gegeben (bis zum Jahr 1854 hatte sich die Zahl der Hydropathen auf 79 erhöht) [39, Vol. 18, 1854, Nr. 4, S. 79], von denen neun von promovierten Ärzten (M.D.) und sechs weitere von jeweils einem «Dr.» geführt wurden [5, S. 96; 41], einschliesslich der «Dr. David Ruggles'schen» Anstalt in Northampton und einer von einem weiteren Laien geführten KWHA. Nach Northampton kam der medizinische Laie Dr. phil. Carl Munde erstmals im März 1850 von New York aus, um sich auf Empfehlung eines Horace Greeley über die Möglichkeiten eines Kaufs des vormaligen «Water-Cure Hospitals» des verstorbenen D. Ruggles zu unterrichten [36, S. 133]. Mundes Freunde liehen ihm das Geld für die erste Anzahlung von 500 USD am 3. Dezember 1851. Zwei weitere Freunde hatten schliesslich eine ordnungsgemässe Übertragung des Besitztums durch eine weitere Zahlung von 1.603,00 USD ermöglicht, und so konnte Munde seine persönliche Erfolgsgeschichte in Sachen Kaltwasserkur in den USA beginnen. Bereits im folgenden Jahr baute er die Anstalt aus, sodass etwa 115 Patienten aufgenommen werden konnten. Hierfür wurde das erste Gebäude der Ruggles'schen «Water-Cure» abgetragen, das andernorts wieder aufgebaut und zunächst durch einen zweistöckigen Bau ersetzt wurde. Bereits 1854 hatte er in Northampton zwei weitere Mitbewerber in Sachen «water-cure» in Form von E.E. Denniston und dem Arzt H. Halsted, M.D., der seine Heilanstalt vom Staat New York nach Northampton, Massachusetts, verlegt hatte [39, Bd. 18, 1854, Nr. 1, S. 18; Nr. 4, S. 79, 114]. 2 Jahre lang arbeitete der in Deutschland 
ausgebildete, in den USA bereits als wissenschaftlich orientiert anerkannte Arzt und Chirurg Dr. J. Rittler in Mundes Anstalt. Dieser leitete anschliessend in Brownsville zusammen mit dem Arzt Dr. C. Baelz eine «Water-Cure»; seit Februar 1855 machte er Werbung damit, dass er an Mundes Anstalt gearbeitet hatte [39, Vol. 19, 1855, Nr. 4, S. 90; Vol. 20, 1855, Nr. 1, S. 17]. Dies ist durchaus als Beleg dafür zu werten, dass Munde einen guten Namen bezüglich der Hydropathie hatte. Munde konnte bis zum Jahr 1856 die Kapazität seiner Anstalt auf 100 Zimmer zur Aufnahme von 150 Patienten erhöhen - mit über $16 \mathrm{Ba}$ deräumen. Aus dem Jahr 1856 existiert noch eine Werbeschrift [42], der im folgenden Jahr eine weitere noch erhaltene, mit geringfügig geänderter Überschrift folgte [43]. In diesen Blättern (jeweils auf einer Seite bzw. 4 Seiten) schildert er die Art seiner hydropathisch-naturheilkundlichen Therapieformen und die hierfür geforderten Beträge, wie er dies mehrfach in Zeitungsannoncen getan hat.

Infolge eines offenen Bekenntnisses zu Abraham Lincoln (1809-1865) und dessen Wahl zum 16. US-Präsidenten gegenüber einer Patientin aus New Orleans verlor Munde innerhalb zweier Tage sämtliche Südstaaten-Patienten, und auch fortan kamen keine mehr zu ihm. Dies führte zwangsläufig zunächst zu einem gewissen Einbruch der Patientenzahlen. Dieses Manko konnte jedoch trotz des sogenannten Sezessionskriegs, des inneramerikanischen Kriegs (1861-1864), bis zum Jahr 1865 mehr als ausgeglichen werden. Da sich Munde offensichtlich inzwischen sogar einen Namen als «Neurologe» gemacht hatte, wie man sich sogar noch 1914 erinnerte [44], hatte er auch neben den üblichen Leiden, wie sie in den KWHAs häufig behandelt wurden, eine zusätzliche Krankenklientel zu erwarten - das waren die «Nervösen». Dies verwundert indes nicht, da auch in den USA bereits frühzeitig Hydropathen, wie etwa im Jahr 1844 der US-Arzt und Hydropath Joel Shew (1816-1855), sowie zahlreiche Vertreter des Trinkens von Mineralwasser glaubten, erkannt zu haben, dass die Ursache aller Krankheiten «der Einfluss einer nervösen Erschöpfung sei» und mit ihren Methoden therapiert werden könne [40, S. 90]. Dieses vielfach beobachtete Erschöpfungsphänomen wurde etliche Jahre später durch den US-Elektrotherapeuten und Neurologen George M. Beard (1839-1883) und viele andere beschrieben [45] und unter anderem als «american nervousness», «neurasthenia americana» oder «americanitis» bezeichnet [40, S. 90]. In Deutschland wurde dieses Krankheitsbild vor allem durch die Arbeiten von Beard/Neisser (1881) und dem Greifswalder Physiologen Rudolf Arndt (18351900) als «Neurasthenie» bekannt gemacht [46]. Dieses Krankheitsbild sollte fortan die Medizin auch hierzulande vor völlig neue Fragen in der Therapie stellen [47], womit sich gerade viele leitende Ärzte in deutschen Wasserheilanstalten zu befassen hatten [2, S. 12]. Mit anderen Wor- ten: Es verwundert nicht, dass Patienten nicht zuletzt aus diesen Gründen fortan auch aus zahlreichen anderen Unionsstaaten in die «Munde's Water-Cure» kamen und sich letztlich die Patientenfrequenz sogar noch bis zu der Zeit nach dem Ende des Sezessionskriegs mit der Rückgliederung der «Amerikanischen Staaten der Konföderierten» (CSA) in die «Vereinigten Staaten von Amerika» (USA) vergrössert hatte. Ein solcher Zuspruch zu den «Wasserheilanstalten» wurde frühzeitig zusätzlich dadurch gefördert, dass man in den USA schon im Laufe der 1850erJahre, vor allem nach dem Ende des Kriegs 1864, zunehmend eine weitere besondere Klientel als potenzielle Interessenten für den Besuch einer KWHA umwarb: die Frauen. Sowohl neurologische als auch typische Frauenkrankheiten nebst Fragen angemessener Kleidung wurden als Spezialität solcher Water-Cures hervorgehoben [40, S. 85]. Dies geht unter anderem aus dem Beispiel der «Highland Home Water-Cure» in Fishkill Landing im Staat New York unter der ärztlichen Leitung von Dr. O.W. May hervor [4, S. 142] (siehe auch WCJ, Vol. 14, 1852, Nr. 3, S. 70/71; Vol. 16, 1853, Nr. 4), der zugleich im August 1852 beschreibt, warum er sich als «Hydropathist» bezeichnete (vgl. WCJ, Vol. 14, 1852, Nr. 2, S. 46); der bereits erwähnte namhafte New Yorker Arzt und Hydropath, Dr. R.T. Trall (1812-1877), war dort zugleich konsultierender Arzt (WCJ, Vol. 19, 1855, Nr. 2, S. 33-34; S. 93) und ein Spezialist in Sachen Frauenkrankheiten. Ein mögliches Indiz für diesen Trend in den Water-Cures ist sicherlich die erwähnte spätere Bezeichnung Mundes als «bekannter Neurologe» - ein Trend, wie er auch in Deutschland anzutreffen war, wo in den Wasserheilanstalten eine zunehmend grössere Zahl von unterschiedlichen Neurosen in der zweiten Hälfte des 19. Jahrhunderts zu behandeln war.

Dann traf Munde am 7. November 1865 ein schwerer Schicksalsschlag. Er verlor seine Anstalt durch ein Feuer, das durch die Unachtsamkeit einer Angestellten ausgebrochen war. Der Totalschaden wurde zwar reguliert und Munde erhielt von seiner Versicherung eine Entschädigungssumme von 125000 USD, jedoch entschied sich Munde gegen einen Wiederaufbau und beendete seine Tätigkeit als «Wasserdoktor» in den Vereinigten Staaten.

Wenngleich die Mundesche Heilanstalt dank ihres Leiters bei den kranken Menschen eine gewisse, jedoch nicht klar zu bemessende Anerkennung erlangt zu haben scheint, wie sich aus der Wiedererreichung der Patientenfrequenz der Vorkriegszeit ergibt, resultierte daraus dennoch keine übermässige, immer wiederkehrende oder häufigere Nennung seines Namens in den einschlägigen hydriatischen Publikationen - von seinen Werbeanzeigen abgesehen. Dennoch bezeichnend für einen gewissen Bekanntheitsgrad, den Munde als Hydriatiker augenscheinlich in den USA erlangen konnte, ist die Tatsache, in welchem Zusammenhang er in einem Bericht von Oscar Handlin über die politische Freiheit in den USA, wie sie 
auch gerade Ausländer wie unter anderem Franzosen oder auch Deutsche vorfanden, genannt wird [48, S. 89]. So wird ausgerechnet Carl Munde neben dem Juristen und «Dreissiger» Gustav Körner (1809-1896) sowie dem vormaligen Bonner Studenten und späteren US-Innenminister (Amtszeit 1877-1881) Carl Schurz (1829-1906), der 1852 in die USA ging, exemplarisch für die Tausende von Emigranten angeführt, welche die neue Freiheit in den USA erfolgreich nutzten [48, S. 93-94]. Dennoch ist davon auszugehen, dass sich aus der Mundeschen Zeit in den USA eingedenk der unterschiedlichen Rahmenbedingungen keine Rückschlüsse dahingehend ziehen lassen, er habe mit seinen dortigen Publikationen oder seiner aktiven Tätigkeit als Leiter einer KWHA auch nur annähernd eine vergleichbare Popularität erreicht, wie ihm dies in seinem Heimatland vorrangig mit seinen Büchern gelungen war.

\section{Munde kehrt mit seiner Familie zurück nach Deutschland}

Obwohl er sich als Persönlichkeit eine ausreichende Reputation zumindest in Northampton erworben hatte und er auch wirtschaftlich trotz des Verlusts seiner Anstalt über eine ausreichende Basis für seine Familie verfügte, hat ihn möglicherweise ein gewisses Heimweh 1866 zur Rückkehr nach Deutschland veranlasst. Trotz seiner wenig erfreulichen politischen Erinnerungen an die alte Heimat war ihm die Rückkehr dank der zwischenzeitlich erfolgten Amnestierung der 1849er und der zusätzlich erworbenen US-Bürgerschaft problemlos möglich. Er kam zunächst kurzzeitig in seine Vaterstadt Freiberg und nach Dresden, um von dort aus über Würzburg nach Cannstatt bei Stuttgart zu ziehen, wo er zeitweilig die Funktion eines US-Konsuls wahrgenommen haben soll [11]. Sein Sohn Paul, der 1866 in Harvard sein Medizinstudium abgeschlossen hatte, blieb nach seiner Teilnahme am 1866erKrieg von 1867 bis 1870 als Assistenzarzt bei dem Gynäkologen Friedrich Wilhelm von Scanzoni (Scanzoni von Lichtenfels) (1821-1891) in Würzburg, um dann als Freiwilliger am deutsch-französischen Krieg als Bataillonsarzt teilzunehmen. Nach Kriegsende wurde Dr. med. Munde junior am 14. März 1871 von seinen Pflichten, zuletzt im Haupt-Feldspital Nr. IV, entbunden [49] und erwarb im Dezember 1871 an der Wiener Universität die Zulassung zur Gynäkologie. Munde junior kehrte im Oktober 1872 nach New York zurück, wo er hochgeachtet als Gynäkologe im Jahr 1902 verstarb. Dessen jüngerer (Halb-)Bruder, der 1880 im österreichischen Görz geborene Dr. Erich Munde, ein Zahnarzt und seit 1911 Mitglied im «Deutschen Liederkranz von New York», der sich im folgenden Jahr für die Stellung der Zahnärzte stark gemacht hatte [50], starb am 27. Februar 1914 im Alter von nur 34 Jah- ren ebenfalls in New York, und zwar als geachteter Spezialist auf dem Gebiet der Anästhesie, wie es in einem Nachruf heisst [44].

In gewisser Weise typisch für Carl G. Mundes unverändert kritische Haltung zur deutschen Obrigkeit ist, dass er kurz nach seiner Rückkehr nach Deutschland bereits im September des Jahres 1868 von Würzburg aus mit einer Publikation in Erscheinung trat, in der es um die Sicherstellung der Rechte von US-Bürgern mit deutscher Herkunft im Norddeutschen Bund ging [15]. Er vertrat die Ansicht, dass die Regierung des Norddeutschen Bundes unter dem Reichskanzler Bismarck diese Bürger im Sinne der eigenstaatlichen Gesetzgebung und unter Missachtung von deren US-Rechten behandelte, was seiner Ansicht nach durch eine Revision des Übereinkommens zwischen dem Norddeutschen Bund und den USA vom 28. Februar 1868 geregelt werden müsse. Der US-Botschafter George Bancroft hatte kurz zuvor eine Intervention zugunsten dieser Bürger davon abhängig gemacht, ob die in Preussen lebenden US-Bürger deutscher Herkunft ihre Steuerzahlungen auch in den USA leisteten [15, Preface]. Das Hauptproblem stellte die Frage dar, unter welchen Umständen naturalisierte Bürger ihre neu erworbene Staatsbürgerschaft wieder verlieren bzw. repatriiert werden konnten und zudem wegen "Verbrechen", die vor 1852 begangen wurden, verfolgt werden konnten - unter Androhung von Auslieferung in das jeweils andere Land $[15$, S. 4]. Ferner ging es darum, wie ein Schutz dieser deutschstämmigen US-Bürger seitens der US-Botschaft gesichert werden könne [15, S. 159]. Gleichzeitig galt es nach Munde zu berücksichtigen, dass viele der Rückwanderer nach Deutschland die USA deshalb verliessen, weil seit dem US-Bürgerkrieg die Kosten in einer Weise gestiegen waren, dass ein Leben für die Familien in Deutschland häufig besser zu bewältigen war [15, S. 157]. So forderte Munde seine US-Mitbürger deutscher Herkunft auf, sich in Clubs usw. zu organisieren, um gegen jegliche «Missstände» aktiv in geeigneter Weise vorgehen und Selbsthilfe leisten zu können. Wenig später leitete Munde selbst in Cannstatt bei Stuttgart zeitweilig ein US-Konsulat [11].

\section{Munde in der Schweiz}

Nachdem Munde 1869 in oberösterreichische Lande umgezogen sein soll, sei er Ende der 1870er-Jahre mit der Leitung der «Wasserheilanstalt» Bad Stein, etwas mehr als $20 \mathrm{~km}$ von Laibach - dem heutigen slowenischen Ljubljana, im damaligen österreichischen Herzogtum Krain gelegen - entfernt, betraut worden [7, S. 34]. Es scheint jedoch, dass Munde von Stuttgart/Cannstatt aus zunächst in die Schweiz übersiedelte, wo er 1874 seine dritte Frau Antonia Heinisch (geb. 06.04.1850), die aus Nürnberg stammte, in Aigle ehelichte (laut Schreiben der US-Ge- 
sandtschaft in Wien an das State Departement vom 30. Juni 1887). Wie aus einigen vagen Angaben Mundes zu entnehmen ist, war er in der Schweiz auch hydropathisch beratend tätig. Jedoch erklärt er im Jahr 1885, das Wetter in der Schweiz habe ihm letztlich nicht so zugesagt. Hingegen beschreibt er die klimatischen Vorzüge der Region um die Stadt Görz am Isonzo, wo er bereits seit 9 Jahren wohne [20, S. 66]. Somit hat Munde etwa 1875/76 die Schweiz mit seiner dritten Ehefrau in Richtung des istrischen Görz (damals noch zu Österreich gehörend; heute: Gorizia/Italien), seines letzten Domizils, wieder verlassen. Mit dieser Frau hatte er zumindest seinen im Jahr 1880 in Görz geborenen Sohn Erich, von dem zum Zeitpunkt der Errichtung seines letzten Testaments im gleichen Jahr noch keine Rede war und der wie erwähnt im Alter von 34 Jahren in den USA gestorben ist. Aus welcher Ehe die drei anderen Kinder stammten, von denen Munde berichtet und die an Diphterie starben sowie in Peuma beigesetzt wurden [20, S. 56], war nicht gesichert zu ermitteln. Möglicherweise hat dieses Drama um seine Kinder ihn zu seiner Schrift über Diphterie und Schlangengift von 1880 veranlasst [20].

\section{Die letzten Jahre}

In einem Passantrag von Antonia Munde vom Mai 1887 beim US-Konsulat (in Triest) ist die Rede von ihrem kleinen sechsjährigen Sohn Erich. Der Fall Antonia Munde, die keine US-Staatsbürgerin war, sondern lediglich mit einem naturalisierten US-Bürger verheiratet und selbst nie in den USA gewesen war, bereitete den US-Behörden (Konsulat in Triest; Gesandtschaft/Botschaft in Wien) ein grosses Problem. Da Frau Munde bei der Antragsstellung selbst bekundet hatte, dass sie zunächst für weitere $15 \mathrm{Jah}$ re in Görz wohnen bleiben wolle, um mit ihrem dann 21-jährigen Sohn in die USA zu ziehen, führte letztlich diese Absichtserklärung nach der Ablehnung des Antrags seitens des Konsulats in Triest zu einer weiteren Prüfung der Angelegenheit durch das «State Departement». Das Aussenministerium sprach sich dann ebenfalls gegen eine Passerteilung an Frau Munde einschliesslich ihres Sohns Erich aus [51, 52].

Im Juli 1882 ist dem Anzeigenblatt der deutschsprachigen Laibacher Zeitung zu entnehmen (Abb. 4), dass in Bad Stein die neu eingerichtete KWHA «abermals unter der ärztlichen Leitung des Dr. Med. Karl Munde», der als «erfahrener Nestor der Hydrotherapie» bezeichnet wird, eröffnet wurde [53], die er dann bis 1884 innehatte [11]. So dürfte Munde als Leiter der Anstalt auch in den Genuss des Besuchs des österreichischen Kaisers Franz Joseph I. gekommen sein, der am 16. Juli 1883 auf der Fahrt durch Bad Stein eine «Allerhöchste Besichtigung der Bade- und Curanstalt» durchführte, woran später eine Gedenktafel
Abb. 4. Ausschnitt «Laibacher Zeitung» vom 11. Juli 1882 über Dr. Mundes Tätigkeit in Bad Stein bei Laibach [53].

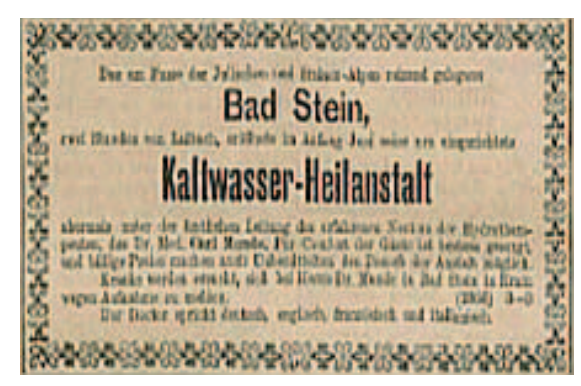

erinnerte [54, S. 47]. Diese Anstalt wird in der «Oesterreichisch-Ungarischen Revue» wegen des Bahnanschlusses als besonders gut erreichbar geschildert [54, S. 233]. Sie existierte als solche noch zur Jahrhundertwende [55].

Eine der letzten Mundeschen Arbeiten erschien 1885 in zweiter Auflage [21], aus der deutlich wird, dass er unverändert bevorzugt nicht nur ein Anhänger der Kaltwasserkuren war, sondern vor allem deren erweiterte Entwicklung innerhalb der Naturheilkunde unterstützte. Abgesehen davon bezeichnete er seine Anstalt unverändert noch als KWHA, obwohl sich zu dieser Zeit üblicherweise die Bezeichnungen «Wasserheilanstalt» oder «Naturheilanstalt» durchgesetzt hatten. Zudem war die klassische Hydropathie im Sinne eines Prießnitz durch die wissenschaftlich begründete Hydro- und Thermotherapie innerhalb der Schulmedizin ergänzt worden. Mit dem bis dahin regional bereits bekannt gewordenen Pfarrer Sebastian Kneipp (1821-1897) in (Bad) Wörishofen erlebte vor allem die naturheilkundlich orientierte Hydrotherapie eine erneute Renaissance jedoch erst nach 1890.

$\mathrm{Ob}$ sich Munde entschloss, wegen der zunehmend schwierigen Lage der Deutschen in der Krain, wie sie unter anderem von Dr. Wilhelm Rohmeder dargelegt wurde [56], seine jeweils nur etwa dreimonatige Tätigkeit in der KWHA in Bad Stein endgültig zu beenden oder lediglich aus Alters- und Gesundheitsgründen und seine letzten Lebensjahre dauerhaft in der istrischen Stadt Görz als Alterssitz zu verbringen, ist nicht belegbar, aber durchaus möglich. Munde zog sich ab 1884 dorthin zurück, wo er inzwischen einen Landsitz inmitten eines grossen Parks erworben hatte. Nachdem ihn sein in New York lebender Sohn Paul bereits im Sommer 1880 in Görz besucht hatte [21, S. 61], kam dieser letztmalig 1886 dorthin [13, S. 193]. Carl Munde soll sein Leben im Alter von 82 Jahren am 08. Februar 1887 in Görz mit einem Pistolenschuss beendet haben [7, S. 34].

\section{Disclosure Statement}

Der Autor erklärt, dass er alleiniger Verfasser dieses Beitrags ist und dieser in keiner anderen Zeitschrift usw. publiziert wurde. Ein Interessenkonflikt besteht nicht. 


\section{Supplemental Material}

Weiterführende Literatur siehe Supplemental Material unter $w w w$.

karger.com/doi/10.1159/000439206.

\section{Literatur}

1 Munde C: Memoiren eines Wasserarztes. Band 1. Dresden/Leipzig, Arnold, 1844. 2. wohlfeilere Ausgabe: Dresden/Leipzig, Arnold, 1847. Band 2, 2. wohlfeilere Ausgabe: Dresden/Leipzig, Arnold, 1847.

2 Averbeck H: Von der Kaltwasserkur bis zur physikalischen Therapie. Betrachtungen zu Personen und zur Zeit der wichtigsten Entwicklungen im 19. Jahrhundert. Bremen, EHV, 2012.

3 Munde C: Hydrotherapie oder die Kunst, die Krankheiten des menschlichen Körpers durch Wasser, Luft, Bewegung und Diät zu heilen und durch eine naturgemäße Lebensweise zu verhüten. Ein Handbuch für Nichtärzte. 9. umgearbeitete und mit einer $\mathrm{Ab}$ handlung über Schwangerschaft, Geburt, Wochenbett und Erziehung der Kinder in der ersten Lebenszeit vermehrte Auflage. Leipzig, Arnoldische Buchhandlung. New York, William Radde, 1853. 10. vermehrte Auflage: Leipzig, Arnoldische Buchhandlung/New York, William Radde, 1861. 11., nach einer dreiunddreißigjährigen Erfahrung und dem heutigen Stande der Wissenschaft gänzlich umgearbeitete Auflage: Leipzig/New York, 1868. 12., vermehrte und verbesserte Auflage: Leipzig, Arnold, 1877. 13. überarbeitete und erweiterte Auflage (Hrsg. Dr. Arthur Pollack): Leipzig, Arnoldische Buchhandlung, 1890.

4 Weiss HB, Kemble H: The Great American Water-cure Craze. A History of Hydropathy in the United States. Trenton/NJ, The Past Times Press, 1967, pp 130-131.

5 The Water-Cure Journal and Herold of Reforms. Vol. VII and VIII. New York, Fowlers and Wells, 1849, pp 186-187.

6 Mitteilung des Stadtarchivs Freiberg an R. Döring vom 08.03.2013; im Besitz des Verfassers.

7 Koch W: Der Freiberger Demokrat Dr. Karl Munde, ein revolutionärer Demokrat von 1848. Die Blende. Heimatblätter für die Kreise Freiberg und Brand-Erbisdorf 1956;2: 32-34.

8 Fege J: Dr. Carl Gottlieb Munde: der Wasserheiler Dr. phil. Dr. med. h.c. Carl Gottlieb Munde (29.3.1805-8.2.1887). Ärzteblatt Sachsen 2009;20:643-644.

9 Fege J: Die medizinische und soziale Betreuung; in 850 Jahre Weißenborn. Vom Waldhufendorf zur Industriegemeinde 1163-2013. Siebenlehn, Druckerei Wagner, 2012, pp 296-298.

10 Merta S: Wege und Irrwege zum modernen Schlankheitskult. Diätkost und Körperkultur nach neuen Lebensstilformen 1880-1930. Stuttgart, Franz Steiner, 2003.

11 Brendel F: Carl Gottlieb Munde. Die Heimatwarte 1939;1: Nr. 3 und Nr. 4, o.S.
12 Wilson CN, Cook SB, Moore A (eds): The Papers of John C(aldwell) Calhoun. Band 27. Colombia, University of South Carolina, 2003, pp 6-9.

13 Munde PF: The Munde watercure; in: Sheffield CA (ed): The History of Florence, Massachusetts. Florence, MA, Selbstverlag, 1895, pp 190-195.

14 Helfricht J: Vincenz Prießnitz (1799-1851) und die Rezeption seiner Hydrotherapie bis 1918. Husum, Matthiesen, 2006.

15 Munde C: The Bancroft Naturalization Treaties with the German States; the United States Constitution and the Rights and Privileges of Citizens of Foreign Birth. Being a Collection of Documents and Opinions Relating to the Subject, to the Encroachment of the NorthGerman Treaty on Our Civil Rights, and the Measures to Rebut It. Würzburg, A. Stuber; New York, William Radde; Philadelphia, Schaefer \& Corradi; St. Louis, C.B. Witter, 1868.

16 Munde C: Genaue Beschreibung der Gräfenberger Wasserheilanstalt und der Priessnitzischen Curmethode. Nebst einer Anweisung über siebzig der am häufigsten vorkommenden Krankheiten, als Gicht, Rheumatismus, Syphilis, Hämorrhoiden, Hypochondrie, Fieber, Entzündungen, Cholera, Influenza und eine Menge anderer chronischer und acuter Uebel durch Anwendung des kalten Wassers mit Schwitzen nach der Gräfenberger Curmethode gründlich zu heilen. Ein Handbuch für diejenigen, die nach Gräfenberg zu gehen oder die Cur zu Hause gesonnen sind sowie für alle Kranken. Nebst einem Anhange über die Behandlung verschiedener Pferdekrankheiten. Leipzig, Hartlebens Verlags-Expedition, 1837.

17 Sauvan L: Darstellung der wissenschaftlichen Prinzipien der Wasserheilkunst oder der sogen. Gräfenberger Heilmethode. Aus dem Französischen übersetzt und mit Zusätzen vermehrt von Dr. Carl Munde. Neisse, T. Hennings, 1840.

18 Der Wasserfreund oder Allgemeine Zeitschrift zur Beförderung der Wasserheilkunde. Hrg. von Doctor Schmitz. Band 1, JanuarJuni. Beilage zu Nr. 41. Erlangen, Ferdinand Enke, 1841, p 2.

19 Munde C: Hydriatic Treatment of Scarlet Fever in its Different Forms. How to Save through a Systematic Application of the Water-Cure, Many Thousands of Lives and Healths, Which Now Annually Perish. New York, William Radde, 1857.

20 Munde C: Diphterie und Schlangengift, Alkohol als Gegengift. Leipzig, Arnoldische Buchhandlung, 1880. Zitiert nach Munde, 1885, p 57.
21 Munde C: Unsere Haut und ihre Beziehungen zu Kaltwasserkuren. Ein Führer zur Erhaltung und Wiederherstellung der Gesundheit ohne Apotheke, namentlich auch bei dem Gebrauch einer Kaltwasserkur in irgendeiner Anstalt, ed 2. Leipzig, Arnoldische Buchhandlung, 1885

22 N.N. [Grafenfeld E]: Der Curgast Deutscher Kaltwasserheilanstalten. Ein Handbuch für alle, welche Kaltwasserheilanstalten gebrauchen und sich über deren Entstehung, Lage, Oertlichkeit, Einrichtung, Frequenz und Erfolge gründlich unterrichten wollen. Leipzig, E.W.B. Naumburg, 1845.

23 Hahn EL: Die heutige Natur- und Wasserheilkunde gegenüber der alten von Prießnitz begründeten Heilmethode, oder die innere Fortentwicklung der Hydriatik. Uebersichtlich dargestellt. Magdeburg, Heinrichshofen'sche Buchhandlung, 1853.

24 Munde C: Hydrotherapie oder die Kunst, die Krankheiten des menschlichen Körpers durch Wasser, Luft, Bewegung und Diät zu heilen. 10. vermehrte Auflage. Leipzig, Arnold/New York, William Radde, 1861, s.a. Nr. 3.

25 Walde, Philo vom: Vinzenz Prießnitz. Sein Leben und Wirken. Prag, Commissionsverlag Fr. A. Urbanek, 1898; Berlin, Wilhelm Möller, 1898, pp 57-58.

26 Lienert M: Naturheilkundiges Dresden. Dresden, Elbhang-Kurier-Verlag, 2002.

27 Munde C: Meine Flucht von Dresden nach New York im Jahre 1849. Gartenlaube 1867; 15: Nr. 10, pp 152-156; Nr. 11, pp 168-171.

28 Wesselhöft JC: Verteidigungsschrift ausgearbeitet von Regierungsrath Schede in Berlin. Für Verwandte und Freunde als Manuscript zum Druck befördert und mit einigen nothwendigen Anmerkungen versehen von Carl Wilhelm Wesselhöft. Jena, den 1. December 1826; in Geheime Verbindungen. Erkenntnis wider die Mitglieder des sogenannten Jünglingsbundes auf dem Grund der zu Cöpnick stattgefundenen Untersuchungen und der hierüber verhandelten Akten gesprochen von dem Königl. Ober-Landesgericht zu Breslau; mit ausdrücklicher Erlaubnis des Königl. Preuß. hohen Ministerii der geistlichen Unterrichts- u. Medicinal-Angelegenheiten verlegt. Band 1. Halle, Eduard Anton, 1826, nach S. 56 beginnend mit S. 1-16. (Hinweis: in diesem Band ist auch eine Antrittsrede von Ober-Medicinalrath Dr. Nepomuk Ringseis «Ueber den revolutionären Geist auf deutschen Universitäten» gehalten am 18 December 1833, enthalten; ed 2. München, 1834.)

29 Schweizer Cl: Johann Wolfgang von Goethe und Kaspar Maria von Sternberg. Naturforscher und Gleichgesinnte. Wien, Lit.-Verlag, 2004, p 250. 
30 Neigebaur JDF: Geschichte der geheimen Verbindungen der neuesten Zeit. 7. Heft. Acten-Stücke über die unter dem Namen des Männer-Bundes und des Jünglings-Bundes bekannten demagogischen Umtriebe (Hrsg: Carl Follenberg). Leipzig, Johann Ambrosius Barth, 1833.

31 Young S: The Wesselhoeft family and homeopathy. www.sueyounghistories.com/archives/ 2007/12/20/the-wesselhoefts-and-hom.

32 Körner G: Das deutsche Element in den Vereinigten Staaten von Nordamerika, 18181848. Cincinnati, A.W. Wilde \& Co., 1880, pp 34-37. Ed 2: New York, E. Steiger \& Co, 1884 pp 176, 432.

33 Sheffield CA (ed): The History of Florence, Massachusetts. Florence, Mass., 1895, p 107.

34 The Eclectic Medical Journal (ed: Buchanan JR). Vol. IV, old series - Vol. II, new series. Cincinnati, W. Phillips \& Co, 1850, p 238.

35 Hodges G, Russell G: David Ruggles. A Radical Abolitionist and the Underground Railroad in New York City. Chapell Hill, University of North Carolina Press, 2010.

36 Porter DB: David Ruggles, 1810-1849: hydropathic practitioner. J Natl Med Assoc 1957; 49:67-72.

37 Porter DB: Early Negro Writing, 1760-1837. (Erstdruck Boston: Beacon Press.) Boston, Black Classic Press, 1995.

38 Munde C: Cold water for scalds, etc. The Water-Cure Journal 1851;XI:92.
39 The Water-Cure Journal and Herold of Reforms; devoted to Physiology, Hydropathy, and the Laws of Life. Vol. XVIII, 1854, Nr. 1 Nr. 4; Vol. XIX, 1855, Nr. 4. New York, Fowlers and Wells.

40 Amstrong D, Metzger Amstrong E: The Great American Medicine Show, Being an Illustrated History of Hucksters, Healers, Health Evangelists, and Heroes from Plymouth Rocks to the Present. New York, Prentice Hall (Simon \& Schuster Inc.), 1991.

41 Whorton JC: Nature Cures. The History of Alternative Medicine in America. New York, Oxford University Press, 2002, pp 89-90.

42 Munde C: Dr. Charles Munde's Water-Cure Establishment at Florence, Mass. Three miles from the Northampton R.R. Depot. Florence, MA, 1856 (Original in: Harvard University, Medical School, Countway Library).

43 Munde C: Regulations for Dr. Charles Munde's Water-Cure Establishment, at Florence (Northampton), Mass. Florence, MA 1857 (Original in: US National Library of Medicine in Bethesda, MD).

44 Obituary [Nachruf] Dr. Erich Munde. The New York Times, 28.02.1914.

45 Beard G. M: Neurasthenia or nervous exhaustion. Boston Med Surg J 1869;5:217-220.

46 Arndt R: Die Neurasthenie (Nervenschwäche), ihr Wesen, ihre Bedeutung und Behandlung vom neurologisch-physiologischen Standpunkte, für Aerzte und Studirende. Wien/Leipzig, Urban \& Schwarzenberg, 1885.
47 Radkau J: Das Zeitalter der Nervosität. Deutschland zwischen Bismarck und Hitler. München, C. Hanser, 1998.

48 Handlin O: The Statue of Liberty. New York, Newsweek, 1971, p 94.

49 Verordnungs-Blatt des Königlich Bayerischen Kriegsministeriums; 1871, Nr. 18. München, FS Hübschmann, 1871

50 Munde E: Still harping my dentor. Oral Hygiene 1912;2:884-886.

51 US Department of State: Foreign Relations of the United States (FRUS). Washington, U.S. Government Printing Office, 1902, p 165.

52 Congressional Edition, Vol. 2532. Washington, U.S. Government Printing Office, 1889, pp XX, 15, 20-23, 1139.

53 Laibacher Zeitung. Anzeigenblatt. 1882, Nr. 156, p 1354.

54 Wasser-Heilanstalt «Bad Stein in Krain». Pfarrer Kneipp'sches Heilverfahren. Stein, Verlag der Curanstalt, ohne Jahr (vermutlich 1898).

55 Rosegger P, et al. (Hrsg), Bürger H (Chefredakteur): Die Südbahn und ihr Verkehrsgebiet in Oesterreich-Ungarn. Brünn/Wien, Rudolf M. Rohrer, 1907, p 108.

56 Rohmeder W: Zur Lage der Deutschen in Krain. Vortrag gehalten im "Vereine zum Schutze deutscher Interessen im Auslande» zu München. München, R. Oldenbourg, 1884. 